\title{
A PROOF OF A CYCLIC VERSION OF DELIGNE'S CONJECTURE VIA CACTI
}

\author{
RaLPh M. KaUfmanN
}

\begin{abstract}
We generalize our results on Deligne's conjecture to prove the statement that the normalized Hochschild co-chains of a finite-dimensional associative algebra with a non-degenerate, symmetric, invariant inner product are an algebra over a chain model of the framed little discs operad which is given by cacti. In particular, in characteristic zero they are a BV algebra up to homotopy and the Hochschild cohomology of such an algebra is a BV algebra whose induced bracket coincides with Gerstenhaber's bracket. To show this, we use a cellular chain model for the framed little disc operad in terms of normalized cacti. This model is given by tensoring our chain model for the little discs operad in terms of spineless cacti with natural chain models for $\left(S^{1}\right)^{\times n}$ adapted to cacti.
\end{abstract}

\section{Introduction}

We prove the following theorem which had been conjectured by D. Tamarkin and B. Tsygan in $[\mathrm{TT}]$

Main Theorem There is chain model for the framed little discs operad which acts naturally on the normalized Hochschild cochains of a finite-dimensional unital associative algebra $A$ with a non-degenerate, symmetric, invariant bi-linear pairing.

The proof which we present below was the first proof of this statement. Our method is to expand our chain model of the little discs operad [K2], which we gave in terms of normalized spineless cacti, to a chain model for the framed little discs operad in terms of normalized cacti. As in [K2], the operation of the cells can be seen as a discretization of the calculations establishing the structure of a BV algebra up to homotopy on the chains of the operad $\mathcal{A} r c$ of [KLP]. The background is the theorem [K1], that the operad of framed little discs is equivalent to the operad of cacti. The Main Theorem can be applied in the situation of string topology [CS1, CS2] to give operations on the loop homology of a simply connected compact manifold. The connection to the $\mathcal{A} r c$ operad puts the whole picture into the context of Moduli spaces and string theory (see also $[\mathrm{K} 4, \mathrm{~K} 5]$ ).

In constructing the cell model, we make use of description of cacti in terms of a bi-crossed product of spineless cacti and an operad built on the monoid $S^{1}$ [K1]. This bi-crossed product is also homotopy equivalent to the semi-direct product of these operads [K1]. For spineless cacti we provided a CW model in terms of normalized spineless cacti [K2] which lead us to give a natural solution to Deligne's conjecture. Now, using the description in terms of the bi-crossed and semi-direct products, we obtain chain models for the operad of framed little discs, by tensoring the chains of normalized spineless cacti with the chains for the operad built on the monoid $S^{1}$. In order to prove the necessary relations on the chain level one can translate the

Received by the editors April 29, 2004. Final version received December 22, 2007. 
respective relations from the relations in the $\mathcal{A} r c$ operad using the method described in $[\mathrm{K} 1, \mathrm{KLP}]$.

As it turns out, in order to translate the relations and thus to establish the homotopy BV structure on the chain level, one needs a refinement of the cell decomposition on the semi-direct product to be able to accommodate all the operations which were used in the $\mathcal{A} r c$ operad picture. This refinement uses cell decompositions on the $S^{1}$ factors which are induced by regarding them as the lobe they represent. This leads to a combinatorial description in terms of planar planted black and white $(\mathrm{b} / \mathrm{w})$ bipartite trees with additional data called spines. In the language of cacti [K1], the additional data keeps track of the position of the local zeros. On these trees, there are linear orders at each vertex, which may differ from the induced linear order of the planar planted trees. This forces us to look at non-rooted trees or equivalently to invert the orientation of the edges. According to the general calculus for "correlation functions" defined by trees, to achieve such an inversion one needs to have a non-degenerate pairing which is symmetric and invariant. This is the assumption we have to make on our algebra. With this assumption, we can rewrite the action of the cellular chains as "operadic correlation functions" for decorated trees. In this description the operation of the chains of the framed little discs operad becomes apparent.

The results and techniques we present below can also be employed in other situations, on which we comment on at the end of the paper. Notably, one can extend the framework to obtain an action of cells of a ribbon graph cell decomposition of moduli space on the $E^{1}$-term of the spectral sequence computing the homology of the loop space of a compact simply connected manifold extending the action of the framed little discs provided by this paper. This leads to string topology like operations of the cells of moduli space of decorated bordered surfaces on the free loop space extending the operations of the string PROP or dioperad.

Assumptions We fix a ground field $k$ of arbitrary characteristic. The algebras we will be considering will be algebras over this ground field. For some results, we will also fix $\operatorname{char}(k)=0$, we will indicate when this is necessary. To avoid any complications, we assume that all the algebras are finite-dimensional. It should be possible to generalize the results below to the situation where $A$ has a bi-linear pairing that induces an isomorphism of $A$ with its dual as an $A$ bi-module, e.g. in a graded situation where all the graded pieces are finite-dimensional.

\section{Background}

2.1. Graphs. In this section, we formally introduce the graphs and the operations on graphs which we will use in our analysis of cacti. This is the approach as given in Appendix B of [K1] in which cacti are characterized as a certain type of ribbon graph. Namely, a cactus is a marked treelike ribbon graph with a metric.

2.1.1. Graphs. A graph $\Gamma$ is a tuple $\left(V_{\Gamma}, F_{\Gamma}, \imath_{\Gamma}: F_{\Gamma} \rightarrow F_{\Gamma}, \partial_{\Gamma}: F_{\Gamma} \rightarrow V_{\Gamma}\right)$ where $\imath_{\Gamma}$ is an involution $\imath_{\Gamma}^{2}=i d$ without fixed points. We call $V_{\Gamma}$ the vertices of $\Gamma$ and $F_{\Gamma}$ the flags of $\Gamma$. The edges $E_{\Gamma}$ of $\Gamma$ are the orbits of the flags under the involution $\imath_{\Gamma}$. A directed edge is an edge together with an order of the two flags which define it. In case there is no risk of confusion, we will drop the subscripts $\Gamma$. Notice that $f \mapsto(f, \imath(f))$ gives a bijection between flags and directed edges. 
We also call $F_{v}(\Gamma):=\partial^{-1}(v) \subset F_{\Gamma}$ the set of flags of the vertex $v$ and call $\left|F_{v}(\Gamma)\right|$ the valence of $v$ and denote it by $\operatorname{val}(v)$. We also let $E(v)=\left\{\{f, \imath(f)\} \mid f \in F_{v}\right\}$ and call these edges the edges incident to $v$.

The geometric realization of a graph is given by considering each flag as a halfedge and gluing the half-edges together using the involution $\imath$. This yields a onedimensional CW complex whose realization we call the realization of the graph.

2.1.2. Trees. A graph is connected if its realization is. A graph is a tree if it is connected and its realization is contractible.

A rooted tree is a pair $\left(\tau, v_{0}\right)$ where $\tau$ is a tree and $v_{0} \in V_{\tau}$ is a distinguished vertex. In a rooted tree there is a natural orientation for edges, in which the edge points toward the root. That is we say $(f, \imath(f))$ is naturally oriented if $\partial(\imath(f))$ is on the unique shortest path from $\partial(f)$ to the root. This means that the set $E(v)$ splits up into incoming and outgoing edges. Given a vertex $v$, we let $|v|$ be the number of incoming edges and call it the arity of $v$. A vertex $v$ is called a leaf if $|v|=0$. Notice that the root is the only vertex for which $\left|v_{0}\right|=\operatorname{val}\left(v_{0}\right)$. For all other vertices $v \neq v_{0}$ one has $|v|=\operatorname{val}(v)-1$.

A bi-colored or black and white (b/w) tree is a tree $\tau$ together with a map $c l r: V \rightarrow$ $\mathbb{Z} / 2 \mathbb{Z}$. Such a tree is called bipartite if for all $f \in F_{\tau}: \operatorname{clr}(\partial(f))+\operatorname{clr}(\partial(\imath(f)))=1$, that is edges are only between black and white vertices. We call the set $V_{w}:=c l r^{-1}(1)$ the white vertices. If $(f, \imath(f))$ is a naturally oriented edge, we call the edge white if $\partial(\imath(f)) \in V_{w}$ and denote the set of white edges by $E_{w}$. Likewise we call $V_{b}:=\operatorname{clr}^{-1}(0)$ the black vertices and let $E_{b}$ be the set of black edges, where a naturally oriented edge $(f, \imath(f))$ is called black if $\partial(\imath(f)) \in V_{b}$.

The black leaves in a rooted black and white tree are called tails. The edges incident to the tails are called tail edges and are denoted $E_{\text {tail }}$. For tails, we will only consider those flags of the tail edges which are not incident to the tail vertices and call them $F_{\text {tail }}$.

2.1.3. Planar trees and Ribbon graphs. A ribbon graph is a connected graph whose vertices are of valence at least two together with a cyclic order of the set of flags of the vertex $v$ for every vertex $v$.

A graph with a cyclic order of the flags at each vertex gives rise to bijections $N_{v}: F_{v} \rightarrow F_{v}$ where $N_{v}(f)$ is the next flag in the cyclic order. Since $F=\amalg F_{v}$ one obtains a map $N: F \rightarrow F$. The orbits of the map $N \circ \imath$ are called the cycles or the boundaries of the graph. These sets have the induced cyclic order.

Notice that each boundary can be seen as a cyclic sequence of directed edges. The directions are as follows. Start with any flag $f$ in the orbit. In the geometric realization go along this half-edge starting from the vertex $\partial(f)$, continue along the second half-edge $\imath(f)$ until you reach the vertex $\partial(\imath(f))$ then continue starting along the flag $N(\imath(f))$ and repeat.

A tree with a cyclic order of the flags at each vertex is called planar. A planar tree has only one cycle $c_{0}$.

2.2. Planar planted trees. A planted planar tree is a rooted planar tree $\left(\tau, v_{0}\right)$ together with a linear order of the set of flags at $v_{0}$. Such a tree has a linear order of all flags as follows: Let $f$ be the smallest element of $\partial^{-1}\left(v_{0}\right)$, then every flag appears in $c_{0}$ and defining the flag $f$ to be the smallest gives a linear order on the set of all flags. 
This linear order induces a linear order on all oriented edges and on all un-oriented edges, by restricting to the edges in the orientation opposite the natural orientation i.e. pointing away from the root. We denote the latter by $\prec$ and its restriction to $E(v)$ or $F(v)$ by $\prec_{v}$.

We will equivalently consider planar planted trees as defined above or as a rooted planar trees whose root vertex has valence one. The bijection in one direction is given by adding a new root vertex and one new edge such that the induced linear structure on the old root is the given one. This tree is called the realization of the planar planted tree. In the other direction the bijection is simply given by contracting the unique edge incident to the root, but retaining the linear order. In the realization of a planar planted tree, we call the unique edge incident to the (new) root $v_{\text {root }}$ the root edge and denote it by $e_{\text {root }}$ and set $f_{\text {root }}$ to be the flag of the root edge which is not incident to the root. Also $E_{\text {root }}=\left\{e_{\text {root }}\right\}, F_{\text {root }}=\left\{f_{\text {root }}\right\}$.

An angle at a vertex $v$ in a planar tree is a pair of two flags incident to $v$ of which one is the immediate successor of the other in the cyclic order of $F_{v}$. There is a bijection between angles, flags and edges by associating to an angle its bigger flag and to the latter the unique edge defined by it.

2.3. The genus of a ribbon graph and its surface. The genus $g(\Gamma)$ of a ribbon graph $\Gamma$ is given by $2 g(\Gamma)+2=\left|V_{\Gamma}\right|-\left|E_{\Gamma}\right|+\#$ cycles.

The surface $\Sigma(\Gamma)$ of a ribbon graph $\Gamma$ is the surface obtained from the realization of $\Gamma$ by thickening the edges to ribbons. I.e. replace each 0 -simplex $v$ by a closed oriented disc $D(v)$ and each 1-simplex $e$ by $e \times I$ oriented in the standard fashion. Now glue the boundaries of $e \times I$ to the appropriate discs in their cyclic order according to the orientations. Notice that the genus of $\Sigma(\Gamma)$ is $g(\Gamma)$ and that $\Gamma$ is naturally embedded as the spine of this surface.

2.3.1. Treelike and marked ribbon graphs. A ribbon graph together with a distinguished cycle $c_{0}$ is called treelike if

i) the graph is of genus 0 and

ii) for all cycles $c_{i} \neq c_{0}$ : if $f \in c_{i}$ then $\imath(f) \in c_{0}$ and if $f \in c_{0}$ then $\imath(f) \in c_{i} \neq c_{0}$.

In other words each edge is traversed exactly once by the cycle $c_{0}$. Therefore there is a cyclic order on all (non-directed) edges, namely the cyclic order of $c_{0}$.

A marked ribbon graph is a ribbon graph together with a map $m k:\{$ cycles $\} \rightarrow F_{\Gamma}$ satisfying the conditions

i) For every cycle $c$ the directed edge $m k(c)$ belongs to the cycle.

ii) All vertices of valence two are in the image of $m k$, that is $\forall v, \operatorname{val}(v)=2$ implies $v \in \operatorname{Im}(\partial \circ m k)$.

Notice that on a marked treelike ribbon graph there is a linear order on each of the cycles $c_{i}$. This order is defined by upgrading the cyclic order to the linear order $\prec_{i}$ in which $m k\left(c_{i}\right)$ is the smallest element.

2.3.2. Dual b/w tree of a marked ribbon graph. Given a marked treelike ribbon graph $\Gamma$, we define its dual tree to be the colored graph whose black vertices are given by $V_{\Gamma}$ and whose set of white vertices is the set of cycles $c_{i}$ of $\Gamma$. The set of flags at $c_{i}$ are the flags $f$ with $f \in c_{i}$ and the set of flags at $v$ are the flags 
$\left\{f: f \in c_{0}, \partial(f)=v\right\}$. The involution is given by $\imath_{\tau}(f)=N(f)$ if $f \in c_{0}$ and $\imath_{\tau}(f)=N^{-1}(f)$ else.

This graph is a tree and is $\mathrm{b} / \mathrm{w}$ and bipartite by construction. It is also planar, since the $c_{i}$ and the sets $F(v)$ have a cyclic order and therefore also $F_{v} \cap c_{0}$. It is furthermore rooted by declaring $\partial\left(m k\left(c_{0}\right)\right)$ to be the root vertex, and declaring $m k\left(c_{0}\right)$ to be the smallest element makes it into a planted tree.

An equivalent definition is given by defining that there is an edge between a pair of a black and a white vertex if and only if the vertex corresponding to $b$ is on the boundary of the cycle $c_{i}$, i.e. $v \in \partial\left(c_{i}\right):=\left\{\partial(f): f \in c_{i}\right\}$.

2.3.3. Spineless marked ribbon graphs. A marked treelike ribbon graph is called spineless, if

i) There is at most one vertex of valence 2 . If there is such a vertex $v_{0}$ then $\partial\left(m k\left(c_{0}\right)\right)=v_{0}$.

ii) The induced linear orders on the $c_{i}$ are compatible with that of $c_{0}$, i.e. $f \prec_{i} f^{\prime}$ if and only if $\imath\left(f^{\prime}\right) \prec_{0} \imath(f)$.

2.3.4. Graphs with a metric. A metric $w_{\Gamma}$ for a graph is a map $E_{\Gamma} \rightarrow \mathbb{R}_{>0}$. The (global) re-scaling of a metric $w$ by $\lambda$ is the metric $\lambda w:(\lambda w)(e)=\lambda w(e)$. The length of a cycle $c$ is the sum of the lengths of its edges length $(c)=\sum_{f \in c} w(\{f, \imath(f)\})$. A metric for a treelike ribbon graph is called normalized if the length of each nondistinguished cycle is 1 .

2.3.5. Marked ribbon graphs with metric and maps of circles. For a marked ribbon graph with a metric, let $c_{i}$ be its cycles, let $\left|c_{i}\right|$ be their image in the realization and let $r_{i}$ be the length of $c_{i}$. Then there are natural maps $\phi_{i}: S^{1} \rightarrow\left|c_{i}\right|$ which map $S^{1}$ onto the cycle by starting at the vertex $v_{i}:=\partial\left(m k\left(c_{i}\right)\right)$ and going around the cycle mapping each point $\theta \in S^{1}$ to the point at distance $\frac{\theta}{2 \pi} r_{i}$ from $v_{i}$ along the cycle $c_{i}$.

2.3.6. Contracting edges. The contraction $\left(\bar{V}_{\Gamma}, \bar{F}_{\Gamma}, \bar{\imath}, \bar{\partial}\right)$ of a graph $\left(V_{\Gamma}, F_{\Gamma}, \imath, \partial\right)$ with respect to an edge $e=\{f, \imath(f)\}$ is defined as follows. Let $\sim$ be the equivalence relation induced by $\partial(f) \sim \partial(\imath(f))$. Then let $\bar{V}_{\Gamma}:=V_{\Gamma} / \sim, \bar{F}_{\Gamma}=F_{\Gamma} \backslash\{f, \imath(f)\}$ and $\bar{\imath}: \bar{F}_{\Gamma} \rightarrow \bar{F}_{\Gamma}, \bar{\partial}: \bar{F}_{\Gamma} \rightarrow \bar{V}_{\Gamma}$ be the induced maps.

For a marked ribbon graph, we define the marking of $\left(\bar{V}_{\Gamma}, \bar{F}_{\Gamma}, \bar{\imath}, \bar{\partial}\right)$ to be $\overline{m k}(\bar{c})=$ $\overline{m k(c)}$ if $m k(c) \notin\{f, \imath(f)\}$ and $\overline{m k}(\bar{c})=\overline{N \circ \imath(m k(c))}$ if $m k(c) \in\{f, \imath(f)\}$, viz. the image of the next flag in the cycle.

2.3.7. Labelling graphs. By a labelling of the edges of a graph $\Gamma$ by a set $S$, we simply mean a map $E_{\Gamma} \rightarrow S$. A labelling of a ribbon graph $\Gamma$ by a set $S$ is a map $L a b\{$ cycles of $\Gamma\} \rightarrow S$, we will write $c_{i}:=L a b^{-1}(i)$. By a labelling of a black and white tree by a set $S$ we mean a map $L a b: E_{w} \rightarrow S$. Again we will write $v_{i}:=L a b^{-1}(i)$.

2.3.8. Planar planted bipartite labelled trees with white leaves. We set $\mathcal{T}_{b p}^{p p, n t}(n)$ to be the set of planar planted bipartite trees which are labelled from $\{1, \ldots, n\}$ with white leaves only. To avoid cluttered notation, we also denote the respective free Abelian group and the $k$-vector space with basis $\mathcal{T}_{b p}^{p p, n t}(n)$ by the same name and let $\mathcal{T}_{b p}^{p p, n t}$ be their union respectively direct sum. 


\subsection{Cacti.}

Definition 2.1. A cactus with $n$ lobes is a $\{0,1, \ldots, n\}$ labelled marked treelike ribbon graph with a metric. I.e. The set $\mathcal{C}$ acti $(n)$ is the set of these graphs. $\mathcal{C}$ act $(n) \subset$ $\mathcal{C}$ acti $(n)$ is the subset of spineless graphs and its elements are called spineless cacti or alternatively cacti without spines. $\mathcal{C}_{a c t i}{ }^{1}(n) \subset \mathcal{C}$ acti $(n)$ is the subset of normalized graphs, called normalized cacti, and finally $\mathcal{C} a c t^{1}(n)=\mathcal{C}$ act $(n) \cap \mathcal{C} a c t i^{1}(n)$ is the set of normalized spineless cacti.

2.4.1. Cactus terminology. The edges of a cactus are traditionally called arcs or segments and the cycles of a cactus are traditionally called lobes. The vertices are sometimes called the marked or special points. Furthermore the distinguished cycle $c_{0}$ is called the outside circle or the perimeter and the vertex $\partial\left(m k\left(c_{0}\right)\right)$ is called the global zero. And the vertices $\partial\left(m k\left(c_{i}\right)\right), i \neq 0$ are called the local zeros. In pictures these are represented by lines rather than fat dots.

Remark 2.2. It is clear that as sets $\mathcal{C}$ acti $(n)=\mathcal{C}$ act $(n) \times\left(S^{1}\right)^{\times n}$ and $\mathcal{C}$ act $(n)=$ $\operatorname{Cact}^{1}(n) \times \mathbb{R}_{>0}^{\times n}$.

For the first statement one notices for each lobe $v_{i}$ there is a unique lowest intersection point $b$ which is the vertex of the outgoing edge of $v$. Thus there is a canonical map $\phi_{i}^{\prime}: S^{1} \rightarrow\left|c_{i}\right|$ which starts at $b$ and goes around the cycle opposite its natural orientation. So to each cycle we associate $\left(\phi_{i}^{\prime}\right)^{-1}\left(\partial\left(m k\left(c_{i}\right)\right)\right)$ that is the co-ordinate of the spine as measured by $\phi_{i}^{\prime}$. This gives the projection onto the factors $\left(S^{1}\right)^{\times n}$. The projection onto the first factor is given by forgetting the spines, i.e. contracting the edges $m k\left(c_{i}\right)$ if $\operatorname{val}\left(\partial\left(m k\left(c_{i}\right)\right)\right)=2$ and changing the marking to the unique marking which makes the graph spineless.

For the second statement the first projection is given by homogeneously scaling the weights of the edges of each non-marked cycle so that their lengths are one. The projection to the factors of $\mathbb{R}_{>0}$ are given by associating to each lobe its length. In both cases the inverse map is clear.

Definition 2.3. The topological type of a spineless cactus in $\mathcal{C} a c t^{1}(n)$ is defined to be its dual b/w tree $\tau \in \mathcal{T}_{b p}^{p p, n t}(n)$.

Remark 2.4. Notice that the arcs of a cactus correspond to the set $E_{\text {arcs }}=E(\tau) \backslash$ $\left(\left\{e_{\text {root }}\right\}\right)$. This bijection can be defined as follows. To a given $e \in E_{\text {arcs }}, e=\{w, b\}$ with $b$ black and $w$ white, we associate the unique arc between the points corresponding to the black vertices $b$ and $b$ - where $b$ - is the black vertex immediately preceding $b$ in the cyclic order of $v$. In other words if $e=\{f, \imath(f)\}$ with $f \in F_{v}$, let $f$ - be the flag immediately preceding $f$ in the cyclic order at $v$, then $b-=\partial(\imath(f-))$. Notice that if $|v|=0$ then and only then $f-=f$.

Remark 2.5. A spineless cactus is uniquely determined by its topological type and the lengths of the segments. 
2.5. The CW complex of normalized spineless cacti. We recall from [K2] the CW complexes $K(n)$. For more details and pictures the reader is referred to [K1, K2].

Remark 2.6. For a normalized spineless cactus the lengths of the arcs have to sum up to the radius of the lobe and the number of arcs on a given lobe represented by a white vertex $v$ is $\operatorname{val}(v)=|v|+1$. Hence the lengths of the arcs lying on the lobe represented by a vertex $v$ are in 1-1 correspondence with points of the simplex $\mid \Delta^{|v|}$. The coordinates of $\left|\Delta^{|v|}\right|$ naturally correspond to the arcs of the lobe represented by $v$ on one hand and on the other hand in the dual $\mathrm{b} / \mathrm{w}$ graph to the edges incident to $v$.

2.5.1. The tree differential in the spineless case. Let $\tau \in \mathcal{T}_{b p}^{p p, n t}$. We set $E_{\text {angle }}=E(\tau) \backslash\left(E_{\text {leaf }}(\tau) \cup\left\{e_{\text {root }}\right\}\right)$ and we denote by num ${ }_{E}: E_{\text {angle }} \rightarrow\{1, \ldots, N\}$ the bijection which is induced by the linear order $\prec^{(\tau, p)}$.

Let $\tau \in \mathcal{T}_{b p}^{p p, n t}, e \in E_{\text {angle }}, e=\{w, b\}$, with $w \in V_{w}$ and $b \in V_{b}$. Let $e-=$ $\{w, b-\}$ be the edge preceding $e$ in the cyclic order $\prec_{w}^{\tau}$ at $w$. Then $\partial_{e}(\tau)$ is defined to be the planar tree obtained by collapsing the angle between the edge $e$ and its predecessor in the cyclic order of $w$ by identifying $b$ with $b-$ and $e$ with $e-$. Formally $w=v_{w h i t e}(e), e-=\prec_{w}^{\tau}(e),\{b-\}=\partial(e-) \cap V_{b}(\tau), V_{\partial_{e}(\tau)}=V(\tau) /(b \sim b-), E_{\partial_{e}(\tau)}=$ $E_{\tau} /(e \sim e-)$. The linear order of $\partial_{e}(\tau)$ is given by keeping the linear order at all vertices which are not equal to $\bar{b}$ where $\bar{b}$ is the image of $b$ and $b-$. For $\bar{b}$ the order is given by extending the linear order $\left(\operatorname{In}(\bar{b}), \prec_{\bar{b}}^{\partial_{e}(\tau)}\right)=\left(\operatorname{In}(b-) \amalg \operatorname{In}(b), \prec_{b-}^{\tau} \amalg \prec_{b}^{\tau}\right)$ - the usual order on the union of totally ordered sets- to $E(\bar{b})$ by declaring the image of $e$ and $e-$ to be the minimal element.

Definition 2.7. We define the operator $\partial$ on the space $\mathcal{T}_{b p}^{p p, n t}$ to be given by the following formula: $\partial(\tau):=\sum_{e \in E_{\text {angle }}}(-1)^{\text {num }_{E}(e)-1} \partial_{e}(\tau)$.

\subsubsection{The Cell Complex.}

Definition 2.8. We define $\mathcal{T}_{b p}^{p p, n t}(n)^{k}$ to be the elements of $\mathcal{T}_{b p}^{p p, n t}(n)$ with $\left|E_{w}\right|=k$.

Definition 2.9. For $\tau \in \mathcal{T}_{b p}^{p p, n t}$ we define $\Delta(\tau):=\times_{v \in V_{w}(\tau)} \Delta^{|v|}$. We define $C(\tau)=$ $|\Delta(\tau)|$. Notice that $\operatorname{dim}(C(\tau))=\left|E_{w}(\tau)\right|$.

Given $\Delta(\tau)$ and a vertex $x$ of any of the constituting simplices of $\Delta(\tau)$ we define the $x$-th face of $C(\tau)$ to be the subset of $|\Delta(\tau)|$ whose points have the $x$-th coordinate equal to zero.

Definition 2.10. We let $K(n)$ be the CW complex whose k-cells are indexed by $\tau \in$ $\mathcal{T}_{b p}^{p p, n t}(n)^{k}$ with the cell $C(\tau)=|\Delta(\tau)|$ and the attaching maps $e_{\tau}$ defined as follows. We identify the $x$-th face of $C(\tau)$ with $C\left(\tau^{\prime}\right)$ where $\tau^{\prime}=\partial_{x}(\tau)$. This corresponds to contracting an edge of the cactus if its weight goes to zero (see Remark 2.4) so that $\Delta(\partial \tau)$ is identified with $\partial(\Delta(\tau))$.

Definition 2.11. We define the topology of $\mathcal{C}_{a c t}{ }^{1}(n)$ to be that induced by the bijection with $K(n)$. Via Remark 2.2 this gives a topology to the spaces $\mathcal{C}$ act $(n), \mathcal{C}$ acti $(n)$ and $\operatorname{Cacti}^{1}(n)$. 


\subsection{The (quasi)-operad structure.}

2.6.1. The operad of cacti. The gluing maps for cacti

$$
\circ_{i}: \mathcal{C} \operatorname{acti}(n) \otimes \mathcal{C} \operatorname{acti}(m) \rightarrow \mathcal{C} \operatorname{acti}(n+m-1)
$$

are defined on elements $\left(c, c^{\prime}\right) \mapsto c \circ_{i} c^{\prime}$ as follows

1) Scaling the weight function $w^{\prime}$ of $c^{\prime}$ by the length $\frac{r_{i}}{R}$ where $r_{i}$ is the length of the cycle $c_{i}$ of the cactus $c$ and $R$ is the length of the cycle $c_{0}$ of $c^{\prime}$.

2) Identifying the realization of the cycle $c_{0}$ of $c^{\prime}$ with the cycle $c_{i}$ of $c$ via the maps $\phi_{0}\left(c^{\prime}\right)$ and $\phi_{i}(c)$, with the orientation on the second $S^{1}$ reversed, as usual.

These maps together with the $\mathbb{S}_{n}$ action permuting the labels turn the collection $\{\mathcal{C}$ acti $(n)\}$ into an operad $\mathcal{C}$ acti. The collection $\{\mathcal{C}$ act $(n)\}$ forms the suboperad $\mathcal{C}$ act.

2.6.2. The quasi-operad of normalized cacti. We recall from [K1] that a quasi-operad is the generalization of a (pseudo)-operad in which the axiom of associativity is omitted and the others are kept.

The gluing maps for normalized cacti

$$
\circ_{i}: \operatorname{Cacti}^{1}(n) \otimes \mathcal{C a c t i}^{1}(m) \rightarrow \mathcal{C a c t i}^{1}(n+m-1)
$$

are defined on elements $\left(c, c^{\prime}\right) \mapsto c \circ_{i} c^{\prime}$ simply by identifying the realization of the cycle $c_{0}$ of $c^{\prime}$ with the cycle $c_{i}$ of $c$ via the maps $\phi_{0}\left(c^{\prime}\right)$ and $\phi_{i}(c)$ again with the orientation on the second $S^{1}$ reversed.

These maps together with the $\mathbb{S}_{n}$ action permuting the labels turn the collection $\left\{\mathcal{C} a c t i^{1}(n)\right\}$ into a homotopy associative quasi-operad $\mathcal{C} a c t i^{1}$. The collection $\left\{\operatorname{Cact}^{1}(n)\right\}$ forms a homotopy associative quasi-suboperad $\mathcal{C a c t}^{1}$ of $\mathcal{C a c t i}^{1}[\mathrm{~K} 1]$.

\subsection{Relations among cacti.}

Theorem 2.12. [K1] Normalized cacti are homotopy equivalent through quasi-operads to the cacti. The same holds for the (quasi)-suboperads of normalized spineless cacti and spineless cacti.

Corollary 2.13. [K1] Normalized cacti are quasi-isomorphic as quasi-operads to cacti and normalized spineless cacti are quasi-isomorphic as quasi-operads to spineless cacti. In particular in both cases the homology quasi-operads are operads and are isomorphic as operads.

2.7.1. Remarks on the bi-crossed product. In this section we recall the construction of the bi-crossed product as it was given in [K1] to which we refer the reader for more details.

First notice that there is an action of $S^{1}$ on $\mathcal{C}$ act $(n)$ given by rotating the base point clockwise (i.e. in the orientation opposite the usual one of $c_{0}$ ) around the perimeter. We denote this action by

$$
\rho^{S^{1}}: S^{1} \times \mathcal{C} a c t(n) \rightarrow \mathcal{C} a c t(n)
$$


With this action we can define the twisted gluing

$$
\begin{aligned}
\circ_{i}^{S^{1}}: \mathcal{C} a c t(n) \times S^{1}(n) \times \mathcal{C} a c t(m) & \rightarrow \mathcal{C} a c t(n+m-1) \\
\left(C, \theta, C^{\prime}\right) & \mapsto C \circ \rho^{S^{1}}\left(\theta_{i}, C^{\prime}\right)=: C \circ_{i}^{\theta_{i}} C^{\prime}
\end{aligned}
$$

Given a cactus without spines $C \in \mathcal{C}$ act $(n)$ the orientation reversed perimeter (i.e. going around the outer circle clockwise i.e. reversing the orientation of the source of $\left.\phi_{0}\right)$ gives a map $\Delta_{C}: S^{1} \rightarrow\left(S^{1}\right)^{n}$.

As one goes around the perimeter the map goes around each circle once and thus the map $\Delta_{C}$ is homotopic to the diagonal $\Delta_{C}\left(S^{1}\right) \sim \Delta\left(S^{1}\right)$.

We can use the map $\Delta_{C}$ to give an action of $S^{1}$ and $\left(S^{1}\right)^{\times n}$.

$$
\rho^{C}: S^{1} \times\left(S^{1}\right)^{\times n} \stackrel{\Delta_{C}}{\longrightarrow}\left(S^{1}\right)^{\times n} \times\left(S^{1}\right)^{\times n} \stackrel{\mu^{n}}{\rightarrow}\left(S^{1}\right)^{\times n}
$$

here $\mu_{n}$ is the diagonal multiplication in $\left(S^{1}\right)^{\times n}$ and $\bar{o}_{i}$ is the operation which forgets the $i$-th factor and shuffles the last $m$ factors to the $i$-th, $\ldots, i+m-1$ st places. Set

$$
\begin{aligned}
\circ_{i}^{C}:\left(S^{1}\right)^{\times n} \times\left(S^{1}\right)^{\times m} \stackrel{\left(i d \times \pi_{i}\right)(\Delta) \times i d}{\longrightarrow}\left(S^{1}\right)^{\times n} \times S^{1} \times\left(S^{1}\right)^{\times m} \\
\stackrel{i d \times \rho^{C}}{\longrightarrow}\left(S^{1}\right)^{\times n} \times\left(S^{1}\right)^{\times m} \stackrel{\bar{o}_{i}}{\longrightarrow}\left(S^{1}\right)^{\times n+m-1}
\end{aligned}
$$

These maps are to be understood as perturbations of the usual maps

$$
\begin{aligned}
& \circ_{i}:\left(S^{1}\right)^{\times n} \times\left(S^{1}\right)^{\times m} \stackrel{\left(i d \times \pi_{i}\right)(\Delta) \times i d}{\longrightarrow}\left(S^{1}\right)^{\times n} \times S^{1} \times\left(S^{1}\right)^{\times m} \\
& \stackrel{i d \times \rho}{\longrightarrow}\left(S^{1}\right)^{\times n} \times\left(S^{1}\right)^{\times m} \stackrel{\bar{o}_{i}}{\longrightarrow}\left(S^{1}\right)^{\times n+m-1}
\end{aligned}
$$

where now $\rho$ is the diagonal action of $S^{1}$ on $\left(S^{1}\right)^{\times n}$. The maps $\circ_{i}$ and the permutation action on the factors give the collection $\left\{\mathcal{S}^{1}(n)\right\}=\left(S^{1}\right)^{\times n}$ the structure of an operad. In fact this is exactly the usual construction of an operad built on a monoid.

Theorem 2.14. [K1] The operad of cacti is the bi-crossed product of the operad $\mathcal{C}$ act of spineless cacti with the operad $\mathcal{S}^{1}$ based on $S^{1}$. Furthermore this bi-crossed product is homotopic to the semi-direct product of the operad of cacti without spines with the circle group $S^{1}$.

$$
\mathcal{C} a c t i \cong \mathcal{C} a c t \bowtie \mathcal{S}^{1} \simeq \mathcal{C} a c t \rtimes \mathcal{S}^{1}
$$

The multiplication in the bi-crossed product is given by

$$
(C, \theta) \circ_{i}\left(C^{\prime}, \theta^{\prime}\right)=\left(C \circ_{i}^{\theta_{i}} C^{\prime}, \theta \circ_{i}^{C^{\prime}} \theta^{\prime}\right)
$$

The multiplication in the semi-direct product is given by

$$
(C, \theta) \circ_{i}\left(C^{\prime}, \theta^{\prime}\right)=\left(C \circ_{i}^{\theta_{i}} C^{\prime}, \theta \circ_{i} \theta^{\prime}\right)
$$

Also, normalized cacti are homotopy equivalent to cacti which are homotopy equivalent to the bi-crossed product of normalized cacti with $\mathcal{S}^{1}$ and the semi-direct product with $\mathcal{S}^{1}$, where all equivalences are as quasi-operads

$$
\mathcal{C} a c t i^{1} \sim \mathcal{C} a c t i \cong \mathcal{C} a c t \bowtie \mathcal{S}^{1} \sim \mathcal{C} a c t^{1} \bowtie \mathcal{S}^{1} \sim \mathcal{C} a c t^{1} \rtimes \mathcal{S}^{1}
$$


Remark 2.15. The proof of the first statement is given by verifying that the two operad structures coincide. For the second statement one notices that the homotopy diagonal is homotopy equivalent to the usual one and that one can find homotopies to the diagonal which continuously depend on the cactus. The third statement follows from contracting the factors $\mathbb{R}_{>0}^{n}$ and using Theorem 2.12 .

Corollary 2.16. The homology operad of $\mathcal{C}$ acti is the semi-direct product of $\mathcal{C}$ acti and the homology of the operad $\mathcal{S}^{1}$ built on the monoid $S^{1}$.

\subsection{Relation to (framed) little discs.}

Theorem 2.17. [K1] The operad $\mathcal{C}$ act is equivalent to the little discs operad and the operad $\mathcal{C}$ acti is equivalent to the framed little discs operad.

The latter result was first claimed by Voronov in [Vor].

\section{A CW decomposition for $\mathcal{C}_{a c t i}{ }^{1}$ and a chain model for the framed little discs}

Definition 3.1. A $\mathbb{Z} / 2 \mathbb{Z}$ decoration for a black and white bipartite tree is a map $\operatorname{dec}^{ \pm}: V_{w} \rightarrow \mathbb{Z} / 2 \mathbb{Z}$.

Proposition 3.2. The quasi-operad of normalized cacti $\mathcal{C}_{\text {acti }}{ }^{1}$ has a $C W$-decomposition which is given by cells indexed by planar planted bi-partite trees with a $\mathbb{Z} / 2 \mathbb{Z}$ decoration. The $k$ cells are indexed by trees with $k-i$ white edges and $i$ vertices marked by 1 .

Moreover cellular chains are a chain model for the framed little discs operad and form an operad. This operad is isomorphic to the semi-direct product of the chain model of the little discs operad given by $C C_{*}(\mathcal{C}$ act $)$ of $[\mathrm{K} 2]$ and the cellular chains of the operad built on the monoid $S^{1}$.

Proof. For the CW decomposition we note that as spaces $\operatorname{Cacti}^{1}(n)=\operatorname{Cact}^{1}(n) \times$ $\left(S^{1}\right)^{\times n}$ see Remark 2.2. Now viewing $S^{1}=[0,1] / 0 \sim 1$ as a 1-cell together with the 0-cell given by $0 \in S^{1}$ the first part of the proposition follows immediately, by viewing the decoration by 1 as indicating the presence of the 1-cell of $S^{1}$ for that labelled component in the product of cells.

To show that the cellular chains indeed form an operad, we use the fact that the bi-crossed product is homotopy equivalent to the semi-direct product in such a way, that the action of a cell $S^{1}$ in the bi-crossed product is homotopic to the diagonal action. This is just the observation that the diagonal and the diagonal defined by a cactus are homotopic. Since a semi-direct product of a monoid with an operad is an operad the statement follows. Alternatively one could just remark, that there is also an obvious functorial map induced by the diagonal for these cells.

The chains are a chain model for the framed little discs operad since $\mathcal{C}_{a c t i}{ }^{1}(n)$ and $\mathcal{C}$ acti $(n)$ are homotopy equivalent and the latter is equivalent to the framed little discs operad. 
Although the above chain model is the one one would expect to use for framed little discs, it does not have enough cells for our purposes. In order to translate the proofs in the arc complex given in [KLP] into statements about the Hochschild complex, we will need a slightly finer cell structure than the one above. After having used the larger structure one can reduce to the cell model with less cells as they are obviously equivalent.

Definition 3.3. A spine decoration $d e c^{\prime}$ for a planted planar bi-partite tree is a $\mathbb{Z} / 2 \mathbb{Z}$ decoration together with the marking of one angle at each vertex labelled by one and a flag at each vertex labelled by zero. We call the set of such trees which are $n$-labelled by $\mathcal{T}_{b p}^{p p, n t, d e c^{\prime}}(n)$ and again use this notation as well for the free Abelian group and the $k$ vector space generated by these sets. We let $\mathcal{T}_{b p}^{p p, n t, d e c^{\prime}}$ be their union respectively direct sum. In pictures we show the angle marking as a line emanating from the vertex which lies between the marked edges and an edge marking by a line through the respective edge. For an example see Figure 1 VI. We sometimes omit the edge marking if the marked edge is the outgoing edge, e.g. in Figure 2.

The realization $\hat{\tau}$ of a planar planted bi-partite tree $\tau$ with a spine decoration is the realization of $\tau$ as a planar planted tree (the root is fixed to be black) together with one additional edge inserted into each marked angle connecting to a new vertex. We call the set of these edges spine edges and denote them by $E_{\text {spine }}$. Likewise set $V_{\text {spine }}$ to be the set of new vertices called the spine vertices which are defined to be black. The spine edges are then white edges. Like for tails, we will only consider the flags of $E_{\text {spine }}$, which are not incident to the spine vertices. We call the set of these flags $F_{\text {spine. }}$. Notice that this tree is the dual tree of a cactus with an explicit marking of the flags $m k\left(c_{i}\right)$. Given a cactus, we call its dual tree with explicit markings its topological type. If $\tau$ had tails, we will split the set of tails of the realization into spines and free tails which are the images of the original tails. $E_{\text {tails }}(\hat{\tau})=E_{\text {ftails }}(\hat{\tau}) \amalg E_{\text {spine }}(\hat{\tau})$ and likewise for the respective flags.

A spine decoration induces a new linear order on the flags incident to the white vertices of its realization. This order $\prec_{v}^{\prime}$ is given by the cyclic order at $v$ and declaring the smallest element to be the spine flag in case $\operatorname{dec}^{ \pm}(v)=1$ and the marked flag in case $\operatorname{dec}^{ \pm}(v)=0$. This gives a canonical identification of $F_{\prec_{v}^{\prime}}: F_{v} \rightarrow\{0, \ldots,|v|\}$.

Proposition 3.4. The spaces $\mathcal{C} a c t i^{1}(n)$ of the quasi-operad of normalized cacti $\mathcal{C}$ act ${ }^{1}$ have $C W$-decompositions $K^{\prime}(n)$ whose cells are indexed by spine decorated planar planted bi-partite trees $\left(\tau\right.$, dec $\left.^{\prime}\right) \in \mathcal{T}_{b p}^{p p, n t, \text { dec }^{\prime}}$ corresponding to the topological type of the cacti. The $k$ cells are indexed by n-labelled trees with $k-i$ white edges and $i$ markings by 1.

Moreover cellular chains of the complex above are a chain model for the framed little discs operad and form an operad.

Proof. The decomposition is almost as in the preceding proposition except that in the product $\mathcal{C}_{\text {act }}{ }^{1}(n) \times\left(S^{1}\right)^{\times n}$ we decompose each factor $S^{1}$ as indicated by the lobe it presents. I.e. for the $S^{1}$ associated to the $n$-th lobe we chose the 0 -cells to be corresponding to the marked points and 1-cells corresponding to the arcs with gluing given by attaching the 1 -cells to the 0 -cells representing the endpoints of the arcs. 
(E.g. 4 0-cells and 4 1-cells for the lobe 1 in Figure $1 \mathrm{VIa}$ ). In terms of trees, the arcs correspond to the angles and thus we take a marking of an arc to be the inclusion of the corresponding 1-cell in the tensor product of the cell complexes. Likewise the edges correspond to the marked points and we take a marking of an edge to be the inclusion of the corresponding 0-cell in the tensor product of the cell complexes.

For the operadic properties, we remark that moving the spine along an arc and then gluing, which is what is parameterized by marking an angle on the lobe $i$ of $c$ when calculating $c \circ_{i} c^{\prime}$, has the effect of moving the base point of $c^{\prime}$ along a complete sequence of arcs until it coincides with a marked point in the composition of the two cacti. This is one side of the bi-crossed product. The effect on the local zeros of $c^{\prime}$ of the movement of the base point is to move them corresponding to structure maps of the bi-crossed product above. The local zeros thus move through a full arc if the global zero passes through the arc on which they lie. Therefore the $\circ_{i}$ product of two cells results in sums of cells. Marking an arc of $c^{\prime}$ obviously gives rise to a sum of cells. Alternatively, one can again just remark that there is a functorial map for the diagonal for this cell model, since there is such a map on the first factor by [K2] and its existence is obvious on the second factor.

The associativity follows from the associativity of cacti. Let $C(\tau), \tau \in \mathcal{T}_{b p}^{p p, n t, d e c^{\prime}}(n)$ be the cells in the CW-complex and $\dot{C}(\tau)$ their interior. Then $P(\tau)=\dot{C}(\tau) \times \mathbb{R}_{>0}^{n}, \tau \in$ $\mathcal{T}_{b p}^{p p, n t, d e c^{\prime}}$ give a pseudo-cell decomposition $\operatorname{Cacti}(n)=\amalg_{\tau} P(\tau)$. It is easy to see that $\operatorname{Im}\left(P(\tau) \circ_{i} P\left(\tau^{\prime}\right)\right)=\amalg_{k} P\left(\tau_{k}\right)$ for some $\tau_{k}$ and $\circ_{i}$ is a bijection onto its image. Let $\circ_{i}^{c o m b}$ be the quasi-operad structure pulled back from $K^{\prime}$ to $\mathcal{T}_{b p}^{p p, n t, d e c^{\prime}}$ and $\circ_{i}^{+}$be the operad structure pulled back from the pseudo-cell decomposition of $\mathcal{C}$ act $i$ to $\mathcal{T}_{b p}^{p p, n t, \text { dec }^{\prime}}$. Then these two operad structures coincide over $\mathbb{Z} / 2 \mathbb{Z}$ thus yielding associativity up to signs. The signs are just given by shuffles, c.f. $\S 4.6$, and are associative as well.

Remark 3.5. Pulling back the operadic compositions, the differential and the grading yields a dg-operad structure on $\mathcal{T}_{b p}^{p p, n t, d e c^{\prime}}$ which is isomorphic to that of

$$
C C_{*}\left(\operatorname{Cacti}^{1}\right):=\bigoplus_{n} C C_{*}\left(K^{\prime}(n)\right)
$$

The operation is briefly as follows: given two trees $\tau, \tau^{\prime} \in \mathcal{T}_{b p}^{p p, n t, d e c^{\prime}}$ the product is $\tau \circ_{i}^{c o m b} \tau^{\prime}=\sum \pm \tau_{k}$ where the $\tau_{k}$ are the trees obtained by the following procedure. Delete $v_{i}$ to obtain an ordered collection of trees $\left(\tau_{l}^{c}, \prec_{v}^{\prime}\right)$ then graft these trees to $\tau^{\prime}$ keeping their order by first identifying the spine edge or marked edge of $v_{i}$ with the root edge of $\tau^{\prime}$ and then grafting the rest of the branches to $\tau^{\prime}$ so that their original order is compatible with that of $\tau^{\prime}$. Lastly contract the image of the root edge of $\tau^{\prime}$ and declare the image of the root of $\tau$ to be the new root. The sign is as explained in 4.6. Due to the isomorphism between $C C_{*}\left(C a c t i^{1}\right)$ and $\mathcal{T}_{b p}^{p p, n t, d e c^{\prime}}$ we will drop the superscript $c o m b$.

3.1. The GBV structure. The picture for the GBV structure is essentially that of [KLP] and goes back to [CS1]. It appears here in another guise, however, since we are now dealing with cells in $C C_{*}\left(C a c t i^{1}\right)$. 

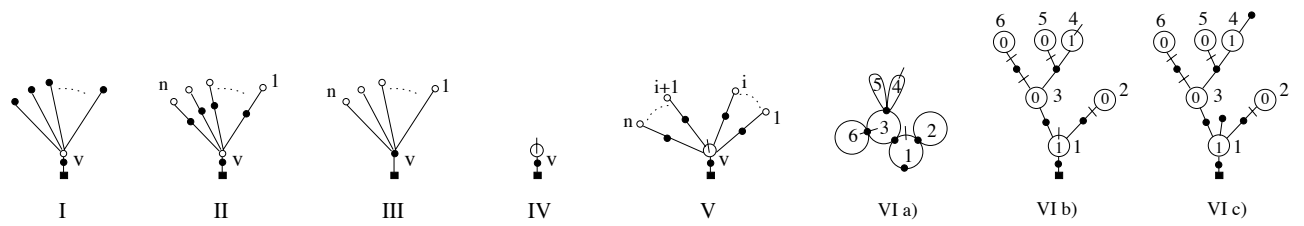

Figure 1. I. the tree $l_{n}$ II. the tree $\tau_{n}$ III. the tree $\tau_{n}^{b}$ IV. the tree O'.V. the tree $\tau_{n, i}^{\prime}$ VI.a) a marked tree-like ribbon graph b) the corresponding decorated tree c) its realization

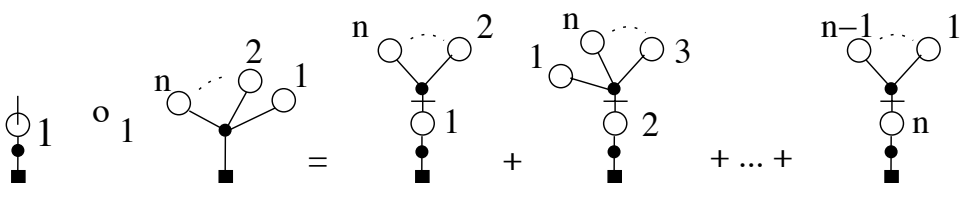

FIgURE 2. The decomposition of the BV operator

First notice that there is a product on the chain level induced by the spineless cactus given by the rooted tree $\tau_{n}$ depicted in Figure 1. Explicitly: $a \cdot b \mapsto \gamma\left(\tau_{2}^{b} ; a, b\right)$ where $\gamma$ is the usual operadic composition. This product gives $C C_{*}\left(C_{a c t i}{ }^{1}\right)$ the structure of an associative algebra with unit. Moreover the product is commutative up to homotopy. The homotopy is given by the usual operation which is induced by $\gamma\left(\tau_{1} ; a, b\right)$. This also induces a bracket which is Gerstenhaber up to homotopy. This can be seen by translating the statements from [KLP, K2], but it also follows from the BV description of the bracket below (Figure 4).

To give the BV structure, let $O^{\prime}$ be the tree with one white vertex, no additional black edges, no free tails and a spine. Notice that the operation $\delta$ induced by $a \mapsto$ $\gamma\left(O^{\prime}, a\right)$ on $C C_{*}\left(C a c t i^{1}\right)$ breaks up on products of chains as follows, see Figure 2

$$
\begin{aligned}
& \delta(a b) \sim \delta(a, b)+(-1)^{|a||b|} \delta(b, a) \\
& \delta(a b c) \sim \delta(a, b, c)+(-1)^{|a|(|b|+|c|)} \delta(b, c, a) \\
&+(-1)^{|c|(|a|+|b|)} \delta(c, a, b) \\
& \delta\left(a_{1} a_{2} \cdots a_{n}\right) \sim \sum_{i=0}^{n-1}(-1)^{\sigma\left(c^{i}, a\right)} \delta\left(a_{c^{i}(1)}, \ldots, a_{c^{i}(n)}\right)
\end{aligned}
$$

where $c$ is the cyclic permutation and $\sigma\left(c^{i}, a\right)$ is the sign of the cyclic permutations of the graded elements $a_{i}$.

Lemma 3.6.

$$
\delta(a, b, c) \sim(-1)^{(|a|+1)|b|} b \delta(a, c)+\delta(a, b) c-\delta(a) b c
$$

Proof. The proof is contained in Figure 3.

Proposition 3.7. The chains $C C_{*}\left(C_{a c t i}{ }^{1}\right)$ are a $G B V$ algebra up to homotopy. That is there is a bracket and a BV operator that satisfy the usual equations up to homotopy. 


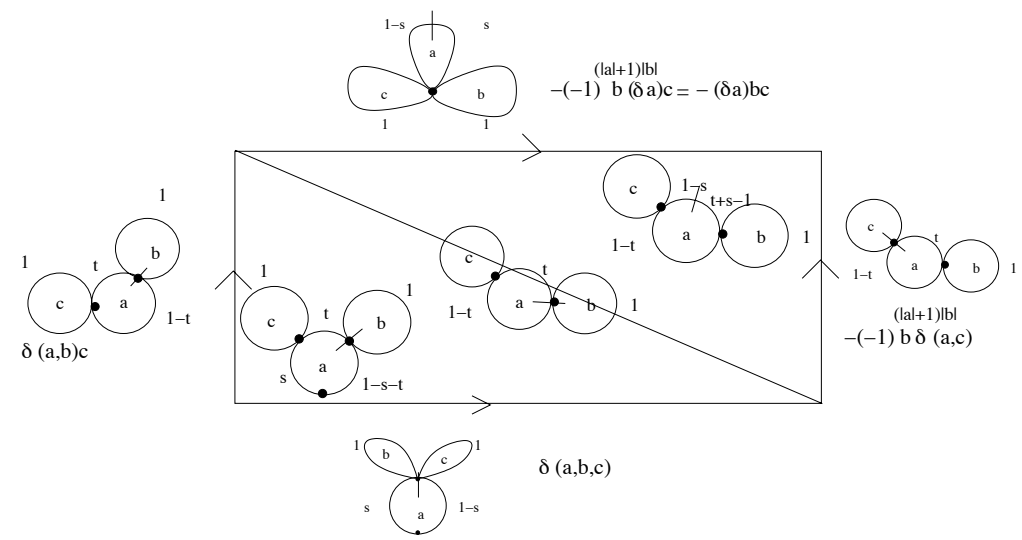

Figure 3. The basic chain homotopy responsible for BV

Taking coefficients in $k$ when $k$ is of characteristic zero, the homology of $\mathcal{C}$ acti hence becomes a $G B V$ algebra.

Proof. The BV structure follows from the Lemma 3.6 via the calculation:

$$
\begin{aligned}
\delta(a b c) \sim & \delta(a, b, c)+(-1)^{|a|(|b|+|c|)} \delta(b, c, a)+(-1)^{|c|(|a|+|b|)} \delta(c, b, a) \\
\sim & (-1)^{(|a|+1)|b|} b \delta(a, c)+\delta(a, b) c-\delta(a) b c+(-1)^{|a|} a \delta(b, c) \\
& +(-1)^{|a||b|} \delta(b, a) c-(-1)^{|a|} a \delta(b) c+(-1)^{(|a|+|b|)|c|} a \delta(b, c) \\
& +(-1)^{|b|(|a|+1 \mid)+|a||c|} b \delta(c, a)-(-1)^{|a|+|b|} a b \delta(c) \\
\sim & \delta(a b) c+(-1)^{|a|} a \delta(b c)+(-1)^{|a+1||b|} b \delta(a c)-\delta(a) b c \\
& -(-1)^{|a|} a \delta(b) c-(-1)^{|a|+|b|} a b \delta(c)
\end{aligned}
$$

Figure 4 contains the homotopy relating the BV operator to the bracket.

\section{The action}

4.1. Assumption. Now we fix $A$ to be a finite-dimensional associative algebra with unit 1 together with an inner product $\eta: A \otimes A \rightarrow k$ which is non-degenerate and both i) invariant: $\eta(a b, c)=\eta(a, b c)$ and ii) symmetric: $\eta(a, b)=\eta(b, a)$. Such an algebra is called a Frobenius algebra.

We will use $C H$ to stand for Hochschild cochains $C H^{n}(A, A):=H o m\left(A^{\otimes n}, A\right)$.

Actually, it would be enough to have a non-degenerate inner-product $\eta$ on $A \simeq$ $C H^{0}(A, A)$ for which i) holds on $H H^{0}(A, A)$, that is up to homotopy for $A$. The condition ii) will then hold automatically up to homotopy since $C H^{0}(A, A)$ is commutative up to homotopy [G].

If one wishes to furthermore relax the other conditions "up to homotopy", one can fix that $\eta$ needs to be non-degenerate only on $H H^{0}(A, A)$ and only require that $H H^{0}(A, A)$ has to be finite-dimensional. In this case, the operadic operations defined below will give operations $f: A^{\otimes n} \rightarrow H H^{0}(A, A)$ and will thus give actions only up to homotopy. This is enough to get the BV structure on $C H^{*}(A, A)$, but not quite 


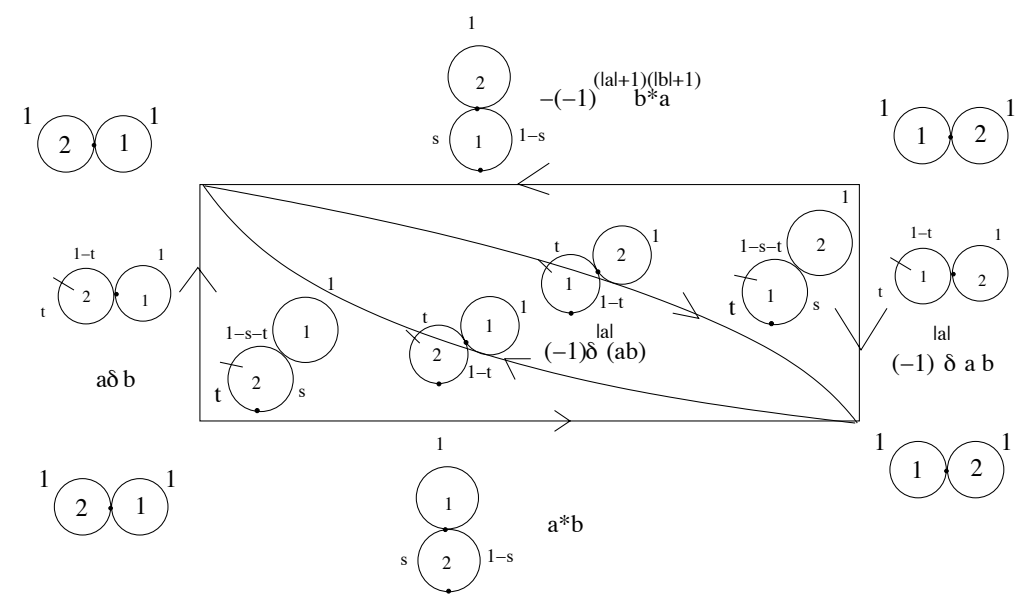

FIGURE 4. The compatibility of the BV operator and the bracket

enough to lift the action to the chain level. We are currently working on such a construction in formal geometry and defer the reader to this upcoming work.

4.2. Notation. Let $\left(e_{i}\right)$ be a basis for $A$ and let $C:=e_{i} \eta^{i j} \otimes e_{j}$ be the Casimir element, i.e. $\eta^{i j}$ is the inverse to $\eta_{i j}=\eta\left(e_{i}, e_{j}\right)$.

With the help of the non-degenerate bilinear form, we identify

$$
C H^{n}(A, A)=H o m\left(A^{\otimes n}, A\right) \cong A \otimes A^{* \otimes n} \cong A^{* \otimes n+1}
$$

We would like to stress the order of the tensor products we choose. This is the order from right to left, which works in such a way that one does not need to permute tensor factors in order to contract.

If $f \in \operatorname{Hom}\left(A^{\otimes n}, A\right)$, we denote by $\tilde{f}$ its image in $A^{* \otimes n+1}$, explicitly $\tilde{f}\left(a_{0}, \ldots, a_{n}\right)=$ $\eta\left(a_{0}, f\left(a_{1}, \ldots, a_{n}\right)\right)$.

With the help of (4.1) we can pull back the Connes' operators $b$ and $B$ (see e.g. $[\mathrm{L}])$ on the spaces $A^{\otimes n}$ to their duals and to $\operatorname{Hom}\left(A^{\otimes n}, A\right)$.

Also let $t: A^{\otimes n} \rightarrow A^{\otimes n}$ be the operator given by performing a cyclic permutation $\left(a_{1}, \ldots, a_{n}\right) \mapsto(-1)^{n-1}\left(a_{n}, a_{1}, \ldots a_{n-1}\right)$ and $N:=1+t+\cdots+t^{n-1}: A^{\otimes n} \rightarrow A^{\otimes n}$.

It is easy to check that the operator induced by $b$ is exactly the Hochschild differential; we will denote this operator by $\partial$. We write $\Delta$ for the operator induced by $B$. It follows that $\Delta^{2}=0$ and $\Delta \partial+\partial \Delta=0$.

4.3. Assumption. To make the formulas simpler we will restrict to normalized Hochschild cochains $\overline{C H}^{n}(A, A)$ which are the $f \in C H^{n}(A, A)$ which vanish when evaluated on any tensor containing $1 \in A$ as a tensor factor (see e.g. [L]). On the normalized chains the operator $\Delta$ is explicitly defined as follows: for $f \in \overline{C H}^{n}(A, A)$

$$
\eta\left(a_{0},(\Delta f)\left(a_{1}, \ldots a_{n-1}\right)\right):=\eta\left(1, f \circ N\left(a_{0}, \ldots a_{n}\right)\right)
$$


4.4. Correlators from decorated trees. We will use the notation of tensor products indexed by arbitrary sets, see e.g. [D]. For a linearly ordered set $I$ denote by $\bigcup_{I} a_{i}$ the product of the $a_{i}$ in the order dictated by $I$.

Definition 4.1. Let $\tau$ be the realization of a spine decorated planted planar $\mathrm{b} / \mathrm{w}$ tree, $v \in V_{w}$, and $f \in \overline{C H}^{|v|}(A, A)$. We define $Y(v, f): A^{F_{v}(\tau)} \rightarrow k$ by

$$
Y(v, f)\left(\bigotimes_{i \in F_{v}(\tau)} a_{i}\right):=\eta\left(a_{F_{\prec_{v}^{\prime}}^{-1}(0)}, f\left(a_{F_{\prec_{v}^{\prime}}^{-1}(1)} \otimes \cdots \otimes a_{F_{\prec_{v}^{\prime}}^{-1}(|v|)}\right)\right)
$$

Set $V_{b-i n t}:=V_{b}(\tau) \backslash\left(V_{\text {tail }} \cup\left\{v_{\text {root }}\right\} \cup V_{\text {spine }}\right)$. For $v \in V_{b-i n t}$ we define $Y(v):=$ $A^{F_{v}(\tau)} \rightarrow k$ by

$$
Y(v)\left(\bigoplus_{i \in F_{v}(\tau)} a_{i}\right)=\eta\left(1, \bigcup_{i \in F_{v}} a_{i}\right)
$$

Definition 4.2. Let $\tau$ be the realization of a planar planted $\mathrm{b} / \mathrm{w}$ tree with $n$ free tails and $k$ labels and $f_{i} \in \overline{C H}^{n_{i}}(A, A)$. For such a tree there is a canonical identification $\left\{v_{\text {root }}\right\} \cup V_{\text {ftail }} \rightarrow\left\{0,1, \ldots,\left|V_{\text {ftail }}\right|\right\}$ which is given by sending $v_{\text {root }}$ to 0 and enumerating the tails in the linear order induced by the planted planar tree. Set $E_{\text {int }}(\tau):=E(\tau) \backslash\left(E_{\text {tail }} \cup E_{\text {root }} \cup E_{\text {spine }}\right)$ and for $\left(a_{0}, \ldots, a_{n}\right) \in A^{\otimes\left(\left\{v_{\text {root }}\right\} \cup V_{\text {ftail }}\right)}$ set

(4.3) $Y(\tau)\left(f_{1}, \ldots, f_{k}\right)\left(a_{0}, \ldots, a_{n}\right):=$

$$
\left[\bigotimes_{v \in V_{w}(\tau)} Y\left(v, f_{L a b(v)}\right) \bigotimes_{v \in V_{b-i n t}} Y_{v}\right]\left[\left(\bigotimes_{i \in F_{\text {ftail }}(\tau) \cup\left\{F_{\text {root }}\right\}} a_{i}\right)\left(\bigotimes_{j \in F_{\text {spine }}} 1\right) \otimes C^{\otimes E_{\text {int }}(\tau)}\right]
$$

In other words, decorate the root flag by $a_{0}$, the free tail flags by $a_{1}, \ldots, a_{n}$, the spines by 1 and the edges by $C$ and then contract tensors according to the decoration at the white vertices while using the product at the black vertices.

Definition 4.3. We extend the definition above by

$$
Y(\tau)\left(f_{1}, \ldots, f_{k}\right)\left(a_{0}, \ldots, a_{n}\right)=0 \text { if }\left|v_{L a b^{-1}(i)}\right| \neq n_{i}=:\left|f_{i}\right|
$$

4.5. The foliage operator. Let $F$ be the foliage operator of [K2] applied to trees. This means that $F(\tau)$ is the formal sum over all trees obtained from $\tau$ by gluing an arbitrary number of free tails to the white vertices. The extra edges are called free tail edges $E_{\text {ftail }}$ and the extra vertices $V_{\text {ftail }}$ are defined to be black and are called free tail vertices.

Using the trees defined in Figure 1 this corresponds to the formal sum $F(\tau):=$ $\sum_{n} l_{n} \circ_{v} \tau$ where the operadic composition is the one for $\mathrm{b} / \mathrm{w}$ trees which are not necessarily bi-partite (see [K2]). In our current setup we should first form $\tilde{F}(\tau):=$ $\sum_{n} \tau_{n} \circ_{v} \tau$ and then delete the images of all leaf edges together with their white vertices of the $\tau_{n}$ to obtain $F(\tau)$. 
4.6. Signs. The best way to fix signs of course is to work with tensors indexed by edges like in $[\mathrm{K} 2, \mathrm{KS}]$. For this one fixes a free object $L$ (free $\mathbb{Z}$-module or $k$-vector space) generated by one element of degree \pm 1 and calculates signs using $L^{\otimes E_{w}(\tau)}$ before applying the foliage operator while using $L^{\otimes E_{\text {weight }}}$ after applying the foliage operator, where $E_{\text {weight }}=E_{w} \cup E_{\text {root }} \cup E_{\text {ftail }} \cup E_{\text {spine }}$.

Explicitly, we fix the signs to be given as follows. For any tree $\tau^{\prime}$ in the linear combination above, we take the sign of $\tau^{\prime}$ to be the sign of the permutation which permutes the set $E_{\text {weight }}$ in the order induced by $\prec$ to the order where at each vertex one first has the root if applicable, then all non-tail edges, then all the free tails, and if there is a spine edge, the spine.

The explicit signs above coincide with usual signs [L] for the operations and the operators $b$ and $B$ and also coincide with the signs of $[\mathrm{G}]$ for the $\circ_{i}$ and hence for the brace operations $[\mathrm{Ge}, \mathrm{Kad}, \mathrm{GV}]$. The signs for the operations corresponding to operations on the Hochschild side are fixed by declaring the symbols "," and "\{" to have degree one.

Definition 4.4. For $\tau \in \mathcal{T}_{b p}^{p p, n t, d e c^{\prime}}$ let $\hat{\tau}$ be its realization. We define the operation of $\tau$ on $\overline{C H}(A, A)$ by

$$
\eta\left(a_{0}, \tau\left(f_{1}, \ldots, f_{n}\right)\left(a_{1}, \ldots, a_{N}\right)\right):=Y(F(\hat{\tau}))\left(f_{1}, \ldots, f_{n}\right)\left(a_{0}, \ldots, a_{N}\right)
$$

Notice that due to Definition 4.3 the right hand side is finite.

4.7. Examples. We will first regard the tree $O^{\prime}$ with one white vertex, no additional black edges, no free tails and a spine, see Figure 1. For a function $f \in \overline{C H}^{n}$ we obtain:

$$
\begin{aligned}
& Y\left(F\left(O^{\prime}\right)\right)(f)\left(a_{0}, \ldots, a_{n-1}\right)=\eta\left(1, f\left(a_{0}, \ldots a_{n-1}\right)\right. \\
& \left.+(-1)^{n-1} f\left(a_{n-1}, a_{0}, \ldots, a_{n-2}\right)+\ldots\right)=\eta\left(a_{0}, \Delta(f)\left(a_{1}, \ldots, a_{n-1}\right)\right)
\end{aligned}
$$

Let $\tau_{n, i}^{\prime}$ be the tree of Figure 1. Then the operation corresponds to $Y\left(F\left(\tau_{n, i}^{\prime}\right)\right)\left(f ; g_{1}, \ldots, g_{n}\right)\left(a_{0}, \ldots, a_{N}\right)=\eta\left(1, f\left\{{ }^{\prime} g_{i+1}, \ldots, g_{n}, g_{1}, \ldots, g_{i}\right\}\left(a_{(2)}, a_{0}, a_{(1)}\right)\right)$ where $N=|f|+\sum\left|g_{i}\right|-n-1$ and we used the short hand notation

$$
\begin{aligned}
& f\left\{g_{j+1}, \ldots, g_{n}, g_{1}, \ldots, g_{j}\right\}\left(a_{(2)}, a_{0}, a_{(1)}\right)=\sum \pm f\left(a_{k+1}, \ldots, a_{i_{j+1}-1},\right. \\
& g_{j+1}\left(a_{i_{j+1}}, \ldots, a_{i_{j+1}+\left|g_{j+1}\right|}\right), \ldots, a_{i_{n}-1}, g_{n}\left(a_{i_{n}}, \ldots, a_{i_{n}+\left|g_{n}\right|} \mid, \ldots, a_{N}, a_{0},\right. \\
& \left.\quad a_{1}, \ldots, a_{i_{1}-1}, g_{1}\left(a_{i_{1}}, \ldots, a_{i_{1}+\left|g_{1}\right|}\right), \ldots, a_{i_{j}-1}, g_{j}\left(a_{i_{j}}, \ldots, a_{i_{j}+\left|g_{j}\right|}\right), \ldots, a_{k}\right)
\end{aligned}
$$

where the sum runs over $1 \leq i_{1} \leq \cdots \leq i_{j} \leq \cdots \leq k \leq \cdots \leq i_{j+1} \leq \cdots \leq i_{n} \leq N$ : $i_{l}+\left|g_{l}\right| \leq i_{l+1}, i_{j}+\left|g_{j}\right| \leq k$ and the signs are as explained above.

Theorem 4.5 (The cyclic Deligne conjecture). The Hochschild cochains of a finitedimensional associative algebra with a non-degenerate, symmetric, invariant, bilinear form are an algebra over the chains of the framed little discs operad. This operation is compatible with the differentials.

Proof. We will use the cellular chains $C C_{*}\left(\operatorname{Cacti}^{1}\right)$ as a model for the chains of the framed little discs operad. It is clear that 4.4 defines an action. On the Hochschild side, the $\circ_{i}$ operations are substitutions of the type $f_{i}=\psi\left(g_{1}, \ldots, g_{n}\right)$. For the chains 
$C C_{*}\left(\operatorname{Cacti}^{1}\right)$ the $\tau \circ_{i} \tau^{\prime}$ operations are the pull-back via the foliage operator of all possible substitutions of elements of $F(\tau), \tau \in C C_{*}\left(\operatorname{Cacti}^{1}\right)$ into the position $i$ of $F\left(\tau^{\prime}\right)$. The action $Y$ then projects onto the substitution $f_{i}=\psi\left(g_{1}, \ldots, g_{n}\right)$ so that the action is operadic. Explicitly the substitution $t \circ_{i}^{s} t^{\prime}$ for planted planar bi-partite trees with a decoration $d e c^{\prime}$ and additional free tails is given as follows: Say the number of tails of $t^{\prime}$ coincides with $\left|F\left(v_{i}\right)\right|$. In this case replace the vertex $v_{i}$ of $t$, its edges and the black vertices corresponding to the edges with the tree $t^{\prime}$ matching the flags of $v_{i}$ with the tails of $t^{\prime}$ by first matching the root edge with the marked flag of $v_{i}$ and then using the linear order. Lastly contract the image of the root flag. Otherwise set $t \circ_{i}^{s} t^{\prime}=0$. With this definition it is easy to see that $F\left(\tau \circ \tau^{\prime}\right)=F(\tau) \circ \circ_{i}^{s} F\left(\tau^{\prime}\right)$.

The compatibility of the Hochschild differential with the differential of the cell complex follows from the relevant statements for $\tau_{n}$ and $\tau_{n}^{b}$, which are straightforward but lengthy calculations (see e.g. [K2, G]), together with the calculations above $\S 4.7$ which are easily modified to show that $\left(\partial O^{\prime}\right)(f)=\Delta(\partial(f))$ and that $\left(\partial \tau_{n, i}^{\prime}\right)\left(f, g_{1}, \ldots, g_{n}\right)=$ $\left(\partial \tau_{n, i}^{\prime}\right)\left(f, g_{1}, \ldots, g_{n}\right) \pm\left(\tau_{n, i}^{\prime}\right)\left(\partial f, g_{1}, \ldots, g_{n}\right)+\sum_{i} \pm\left(\tau_{n, i}^{\prime}\right)\left(f, g_{1}, \ldots, \partial\left(g_{i}\right), \ldots, g_{n}\right)$ via an even more lengthy but still straightforward calculation. This then verifies the claim in view of the compatibility of the differentials and the respective operad structures.

Alternatively, in view of the operation of the foliage operator, the compatibilities follow from a straightforward translation of trees with tails into operations on the Hochschild complex. The compatibility of the differential then follows from the almost identical definition of the differential for trees with tails of [K2] and that in the Hochschild complex as $\partial(f)=f \circ \cup-(-1)^{|f| \mid} \cup \circ f$.

Corollary 4.6. The normalized Hochschild cochains of an algebra as above are a GBV algebra up to homotopy in the sense of Proposition 3.7.

This could of course have been checked directly without recourse to the operation of a chain model, but we do not know of any source for this result. It also seems to be difficult to guess the right homotopies as Gerstenhaber did in the non-cyclic case $[G]$. The content of the next corollary was expected $[\mathrm{C}]$, but we again could not find a source for it.

Corollary 4.7. Over a field of characteristic zero, the Hochschild cohomology of an algebra as above is a BV algebra, such that the induced bracket is the Gerstenhaber bracket.

Lastly, since our second version of cellular chains of Proposition 3.4 are a subdivision of the cell decomposition of Proposition 3.2, we can also use the latter cell decomposition.

Corollary 4.8. The normalized Hochschild cochains of an algebra as above are an algebra over the semi-direct product over a chain model of the little discs operad and a chain model for the operad $\mathcal{S}$ built on the monoid $S^{1}$.

Remark 4.9. The operation of the little discs operad by braces, viz. the original Deligne conjecture as discussed in [K2] for Frobenius algebras, corresponds to the decorations in which $d e c^{ \pm} \equiv 0$ and the decorated edge is always the outgoing edge.

Remark 4.10. In the Theorem 4.5 we can relax the conditions and implications as explained in $\S 4.1$. 


\section{Relation to String Topology and Variations}

5.1. String Topology like operations. Let $M$ be a simply connected compact manifold $M$ and denote the free loop space by $\mathcal{L} M$ and let $C_{*}(M)$ and $C^{*}(M)$ be the singular chains and co-chains of $M$. We know from $[\mathrm{J}, \mathrm{CJ}]$ that $C_{*}(\mathcal{L} M) \simeq$ $C H^{*}\left(C^{*}\left(M, C_{*}(M)\right)\right.$ and $H_{*}(\mathcal{L} M) \simeq H H^{*}\left(C^{*}(M), C_{*}(M)\right)$. Moreover $C^{*}(M)$ is an associative $d g$ algebra with unit, differential $d$ and an integral $(M$ was taken to be a compact manifold) $\int: C^{*}(M) \rightarrow k$ such that $\int d \omega=0$ for $\omega \in C^{*}(M)$. The integral descends to $H:=H^{*}(M)$ and makes it into a Frobenius algebra. By using the spectral sequence and taking field coefficients we obtain an action on $E^{1}=$ $C H^{*}(H, H)$ compatible with the differential, that hence descends to the subsequent terms in the sequence which ultimately converges to $H H^{*}\left(C^{*}(M)\right)$ and hence induces an operadic action on the level of (co)-homology.

Corollary 5.1. When taking field coefficients, the above action gives a dg action of a dg-operad of a chain model of the famed little discs on the $E^{1}$-term of a spectral sequence converging to $H_{*}(L M)$. This induces an operation on the homology of the loop space of a simply connected compact manifold making it into a BV algebra.

Remark 5.2. We know from [CJ, Me] that the induced multiplication is indeed the multiplication for string topology.

5.2. Variation, Generalizations and the Connection to Moduli Spaces. There are generalizations of the constructions above to moduli space which are very subtle and involved [K4, K5]. The connection is made via the map called loop of [KLP] which maps the so-called $\mathcal{A} r c$ operad to ribbon graphs with marked points on the cycles of the graph. In the case of no punctures the analysis of this map in terms of Strebel differentials yields an isomorphism between the moduli space $M_{g, n}^{1^{n}}$ of genus $g$ surfaces with $n$ marked points and a tangent vector at each of these points [K4] and the suboperad of quasi-filling arcs. The latter is also homotopy equivalent to the moduli space of decorated bordered surfaces $[\mathrm{P}]$. In this way one obtains a cell decomposition of the aforementioned moduli space $M_{g, n}^{1^{n}}$ in terms of marked ribbon graphs. Moreover the correspondence induces an operadic structure on the ribbon graphs by pulling back the gluings from the $\mathcal{A} r c$ operad. Using the notion of operadic correlation functions one can obtain an action of the cells on the cyclic complex. Moreover, a further decoration of the cells by $\mathbb{Z} / 2 \mathbb{Z}$ produces an operad which acts on the cyclic complex of such an algebra and is compatible with the differential when passing to an associated graded [K5]. When restricted to the part corresponding to the little discs the action corresponds to the one given in [MS], in which the authors also announced a generalization to the cyclic case.

Finally, one can define a topological quasi-PROP which has a $\mathrm{CW}$ model whose chains are a dg-PROP of Sullivan Chord diagrams and let it act in a dg-fashion on the Hochschild co-chain complex of a Frobenius algebra [K5]. Similar results using a different version of Sullivan-Chord diagrams have been obtained in [TZ], after the results of this paper were posted.

It is to be expected that there are $A_{\infty}$ - versions of all the these statements which can be deduced from a conjectural "blow-up" of the cacti operads first presented in [K3]. Here the cells are given by products of associahedra and cyclohedra and are 
indexed by trees of the type appearing in [KS]. The details of the action for the little discs will be contained in [KSch].

\section{Acknowledgments}

We would like to thank Alain Connes for an enlightening discussion and Jim Stasheff for his valuable comments. We also thank the Max-Planck-Institute for Mathematics in Bonn for providing the atmosphere and stimulus to conceptualize and complete this paper.

\section{References}

[C] A. Connes, Private communication.

[D] P. Deligne, Catégories tannakiennes, The Grothendieck Festschrift, Vol. II, 111-195,

[CS1] M. Chas and D. Sullivan, String Topology, Preprint math.GT/9911159 To appear in Ann. of Math.

[CS2] _ Closed string operators in topology leading to Lie bialgebras and higher string algebra, in: The legacy of Niels Henrik Abel, 771-784, Springer, Berlin, 2004.

[CJ] R. L. Cohen and J.D.S. Jones, A homotopy theoretic realization of string topology, Math. Ann. 324 (2002), no. 4, 773-798.

[G] M. Gerstenhaber, The cohomology structure of an associative ring, Ann. of Math. 78 (1963), 267-288.

[Ge] E. Getzler, Cartan homotopy formulas and the Gauss-Manin connection in cyclic homology, Quantum deformations of algebras and their representations (Ramat-Gan, 1991/1992; Rehovot, 1991/1992), 65-78, Israel Math. Conf. Proc., 7, Bar-Ilan Univ., Ramat Gan, 1993.

[GV] A. A. Voronov and M. Gerstenkhaber, Higher-order operations on the Hochschild complex, (Russian) Funktsional. Anal. i Prilozhen. 29 (1995), no. 1, 1-6, 96; translation in Funct. Anal. Appl. 29 (1995)

[J] J.D.S. Jones, Cyclic homology and equivariant homology, Inventionnes Math. 87 (1987), 403423

[Kad] T. Kadeishvili, The structure of the $A(\infty)$-algebra, and the Hochschild and Harrison cohomologies, Trudy Tbiliss. Mat. Inst. Razmadze Akad. Nauk Gruzin. SSR 91 (1988).

[K1] R. M. Kaufmann, On several varieties of cacti and their relations, Algebraic \& Geometric Topology, 5 (2005), 237-300.

[K2] _ On Spineless Cacti, Deligne's Conjecture and Connes-Kreimer's Hopf Algebra, in: N. Tongring and R. C. Penner "Woods Hole Mathematics. Perspectives in Mathematics and Physics", Series on Knots and Everything - Vol. 34, World Scientific 2004.

[K3] _ Operads, Moduli of Surfaces and Quantum Algebras, in "Wood's Hole Mathematical Meetings", World Scientific. in: N. Tongring and R. C. Penner "Woods Hole Mathematics. Perspectives in Mathematics and Physics", Series on Knots and Everything - Vol. 34, World Scientific 2004

[K4] — Moduli space actions on the Hochschild cochain complex I: cell models, Journal of Noncommutative Geometry 1, 3 (2007) 333-384.

[K5] _ Moduli space actions on the Hochschild cochain complex II: correlators, Journal of Noncommutative Geometry, 2 (2008), 283-332.

[KLP] R. M. Kaufmann, M. Livernet and R. B. Penner, Arc Operads and Arc Algebras, Geometry and Topology, 7 (2003), 511-568.

[KS] M. Kontsevich and Y. Soibelman, Deformations of algebras over operads and Deligne's conjecture, Conférence Moshé Flato 1999, Vol. I (Dijon), 255-307, Math. Phys. Stud., 21, Kluwer Acad. Publ., Dordrecht, 2000.

[KSch] R. M. Kaufmann and R. Schwell, Cyclohedra, Associahedra and the $A_{\infty}$ Deligne conjecture, In preparation.

[L] J-L. Loday, Cyclic homology, Appendix E by María O. Ronco. Second edition. Chapter 13 by the author in collaboration with Teimuraz Pirashvili. Grundlehren der Mathematischen Wissenschaften , 301. Springer-Verlag, Berlin, 1998. 
[MS] J. E. McClure and J. H. Smith, Operads and cosimplicial objects: an introduction, in: Axiomatic, enriched and motivic homotopy theory, 133-171, NATO Sci. Ser. II Math. Phys. Chem., 131, Kluwer Acad. Publ., Dordrecht, 2004.

[Me] S. A. Merkulov, De Rham model for string topology, Int. Math. Res. Not. (2004), no. 55, 2955-2981.

[P] R. C. Penner, Decorated Teichmüller theory of bordered surfaces, Comm. Anal. Geom. 12 (2004), no. 4, 793-820.

[TT] D. Tamarkin and B. Tsygan, Noncommutative differential calculus, homotopy BV algebras and formality conjectures, Methods Funct. Anal. Topology 6 (2000), no. 2, 85-100.

[TZ] T. Tradler and M. Zeinalian, On the Cyclic Deligne Conjecture, J. Pure Appl. Algebra 204 (2006), no. 2, 280-299.

[Vor] A. A. Voronov, Notes on universal algebra, Graphs and Patterns in Mathematics and Theoretical Physics (M. Lyubich and L. Takhtajan, eds.), Proc. Sympos. Pure Math. 73 (2005), $81-103$.

Purdue University, Department of Mathematics, 150 N. University St, West Lafayette, IN 47907-2067

E-mail address: rkaufman@math.purdue.edu 